\title{
Development of MEMS Inertial Sensors at NUST
}

\author{
Anping Qiu, Yan Su, Qin Shi, and Guoming Xia \\ School of Mechanical Engineering, Nanjing University of Science and Technology, Nanjing, \\ 210094, China
}

Keywords: Inertial sensors, MEMS, vibratory gyroscope, resonant accelerometer.

\begin{abstract}
MEMS Inertial Technology Research Center at Nanjing University of Science and Technology(NUST) has been working on MEMS vibratory gyroscope and MEMS accelerometers for over 15 years, and now dedicates for higher tactical grade MEMS vibratory gyroscope and inertial grade MEMS resonant accelerometer(hereinafter referred to as SiVG and SRA respectively).

This paper presents an overview of the operational principles, fabrication, signal processing and recent developments of the performance of the SiVG and SRA at NUST.
\end{abstract}

\section{Introduction}

The SiVG operates on the Coriolis Effect principle that converts momentum of vibrating objects into a force proportionate to the angular rate of the plane of vibration. The SiVG with a measurement range of $\pm 300 \mathrm{deg} / \mathrm{s}$ and bias stability $(1 \sigma)$ of $10 \mathrm{deg} / \mathrm{hr}$ has been batch production, and largely used in the attitude stabilization system. Under room temperature conditions and within measurement range of $\pm 300 \mathrm{deg} / \mathrm{s}$, the bias stability $(1 \sigma)$ of the improved $\mathrm{SiVG}$ is quite close to $1 \mathrm{deg} / \mathrm{hr}$.

The SRA belongs to the generic category of accelerometers known as VBAs, which sense acceleration by measuring the change in the resonant frequency of beam oscillators under the inertial loading of a proof mass. The bias stability $(1 \sigma)$ of the SRA is close to 5.0 $\mu$ g for an hour, and thereby the bias-instability computed about $0.5 \mu \mathrm{g}$ under room temperature conditions.

This paper describes the development of NUST's MEMS inertial sensors. The performance of MEMS inertial sensors will be presented.

\section{SiVG}

Operational Principle. The SiVG sensor element is shown in Fig. 1. The gyro consists of two proof masses supported by a system of suspension elements. The drive elements are used for electrostatically driving the proof masses antiparallel to the substrate in the drive axis (x-axis).

When the device is rotated about the out-of-plane z-axis, the Coriolis acceleration will deflects the proof masses in the sense axis (y-axis) parallel to the substrate by an amount proportional to the product of the input rotational rate and the drive velocity.

The SiVG die is approximately $4.2 \mathrm{~mm} \times 3.5 \mathrm{~mm}$ in size, and the drive frequency is about $6-10 \mathrm{kHz}$.

The SiVG operated under split-mode condition. The working modes are separated from other natural frequencies of the structure to avoid spurious rate output and the lowest natural frequency is well above external vibration input giving good shock and vibration immunity. The mechanical sensitivity is on the order of 10-4 micro meter under $1 \mathrm{deg} / \mathrm{s}$.

Differential proof mass motion induces electrical currents in the structure that flow through the suspensions and sense pickoff into a preamplifier whose input contains the input angular rate modulated by the drive frequency. Subsequent signal processing converts the signal amplitude to the angular rate magnitude.

Die Process and Vacuum Packaging. The SiVG die is fabricated with SOI processing. The process cross-section is schematically represented in Fig. 2. The die is realized with three wafers, the substrate, the SOI device layer and the cover. The device layer is $80 \mu \mathrm{m}$-thick and manufactured using Deep Reactive Ion Etching on SOI wafer with a high aspect ratio, up to 1:30. The cover and the active SOI layer are joined by $\mathrm{Au} / \mathrm{Si}$ eutectic bonding, forming a hermetic cavity which 
maintains the vacuum needed for high $\mathrm{Q}$ operation of the gyro. In order to maintain the vacuum level over the long term, a getter is adhered to the inner surface of the cover, and once activated the getter progressively absorbs and traps gaseous species. Fig. 3 shows the wafer-level vacuum packaged SiVG dies.

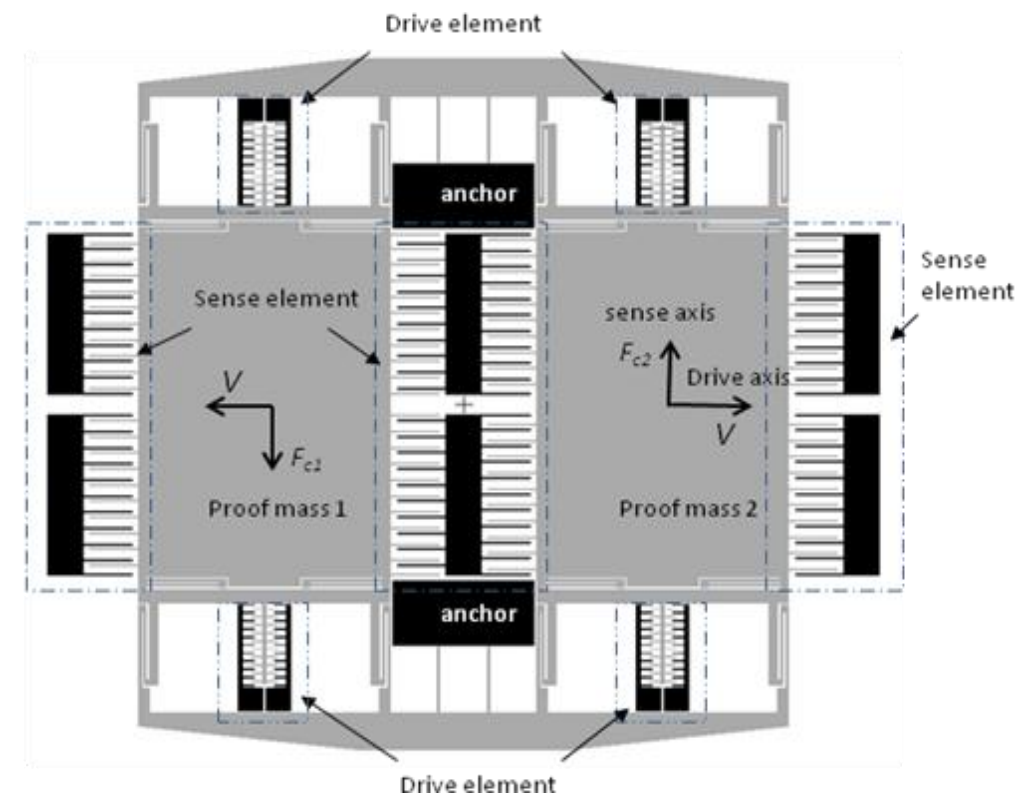

Fig.1. Schematic drawing of the SiVG.
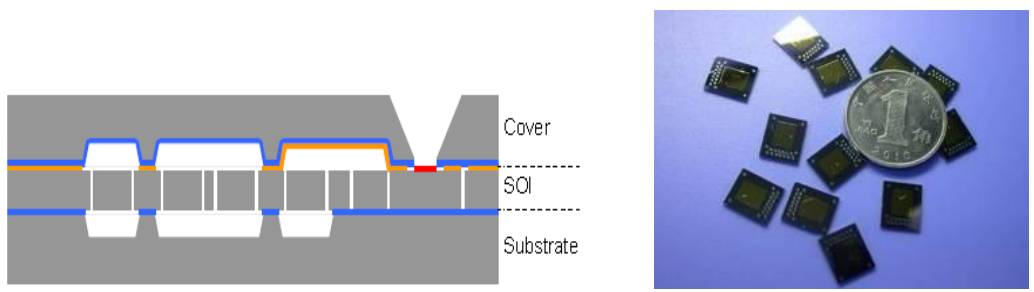

Fig. 2. Process cross section of the die. Fig. 3 Wafer-level vacuum packaged dies.

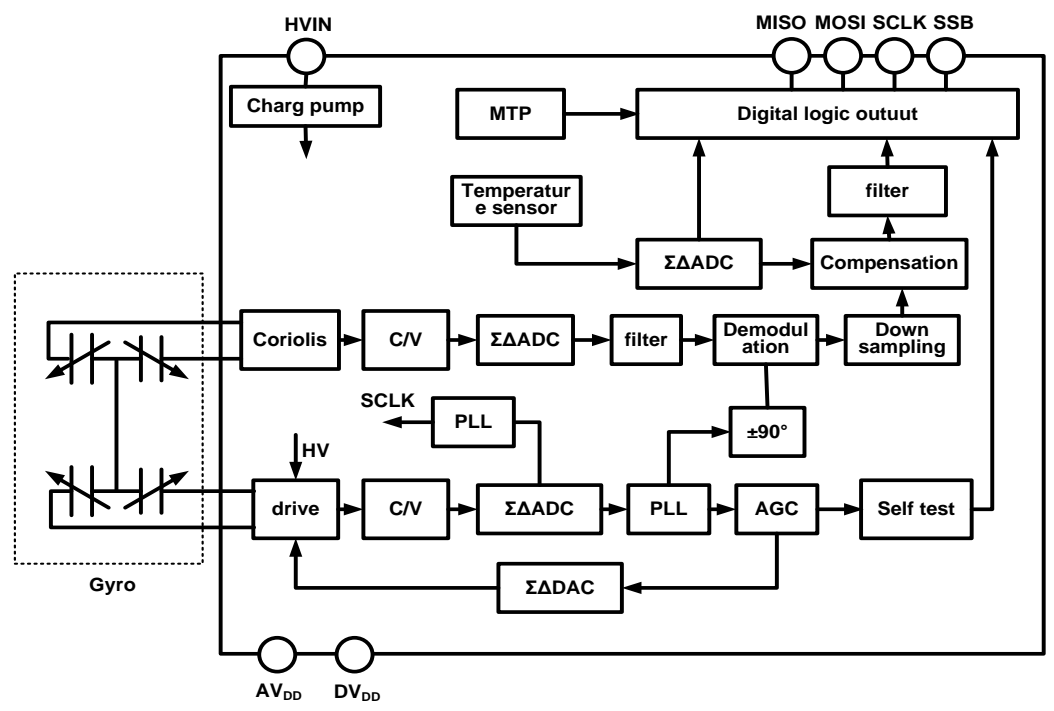

Fig. 4. Schematic diagram of SiVG signal processing.

Signal Processing. The signal processing in the SiVG is based upon two main loops, as shown in Fig. 4. The drive loop is a positive feedback loop with automatic gain control (AGC), which keeps the drive mode of the gyro oscillating at its natural frequency, at constant amplitude. The sense loop has an open-loop operation, and thus the frequency separation determines the bandwidth 
of the SiVG. The digital signal processing provides a stable, high-resolution implementation of the loop filters and sigma-delta DACs, that makes a solid foundation for the high performance SiVG. The signal processing of the SiVG is a $10 \mathrm{~mm}^{2}$ chip manufactured with a $0.18 \mu \mathrm{m}$ CMOS.

Assembly and Packaging. Both wafer-level vacuum packaged SiVG and IC dies are stacked and glued to a ceramic package as it provides perfect hermeticity, and thereby excellent long-term environmental protection for the silicon dies. Wire-bonding are used to connect the MEMS and IC dies together and also the IC die to the Ceramic Leadless Chip Carrier package shown in Fig.5. The SiVG is about $11.4 \times 11.4 \times 3.8 \mathrm{~mm}^{3}$. The assembly has a major influence on the performance of the device, especially with respect to the drift and the scale factor stabilities. Hence reducing levels of transmitted stress and strain to the entire MEMS die is one of the key elements to improve the thermal gyro performances.

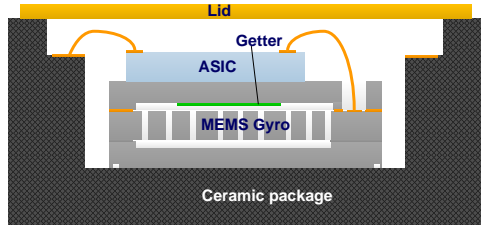

(a)

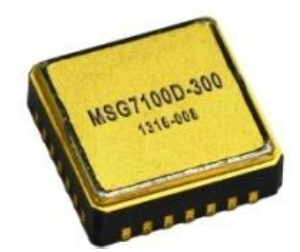

(b)

Fig.5 (a) Schematic of laminated assembly. $\quad$ (b) View of the SiVG.

Performance Testing. Fig. 6 shows a typical SiVG zero-rate output curve at $1 \mathrm{~Hz}$ sample rate for 1 hour under room temperature condition, which the measured bias stability $(1 \sigma)$ is $2.4 \mathrm{deg} / \mathrm{hr}$ in this case. Fig.7 shows the Allan variance curve computed from the raw data shown in Fig.6. The coefficients of angle random walk (ARW) and bias instability are achieved using the least squares method, $0.07 \mathrm{deg} / \sqrt{ } \mathrm{hr}$ and $0.99 \mathrm{deg} / \mathrm{hr}$ respectively.
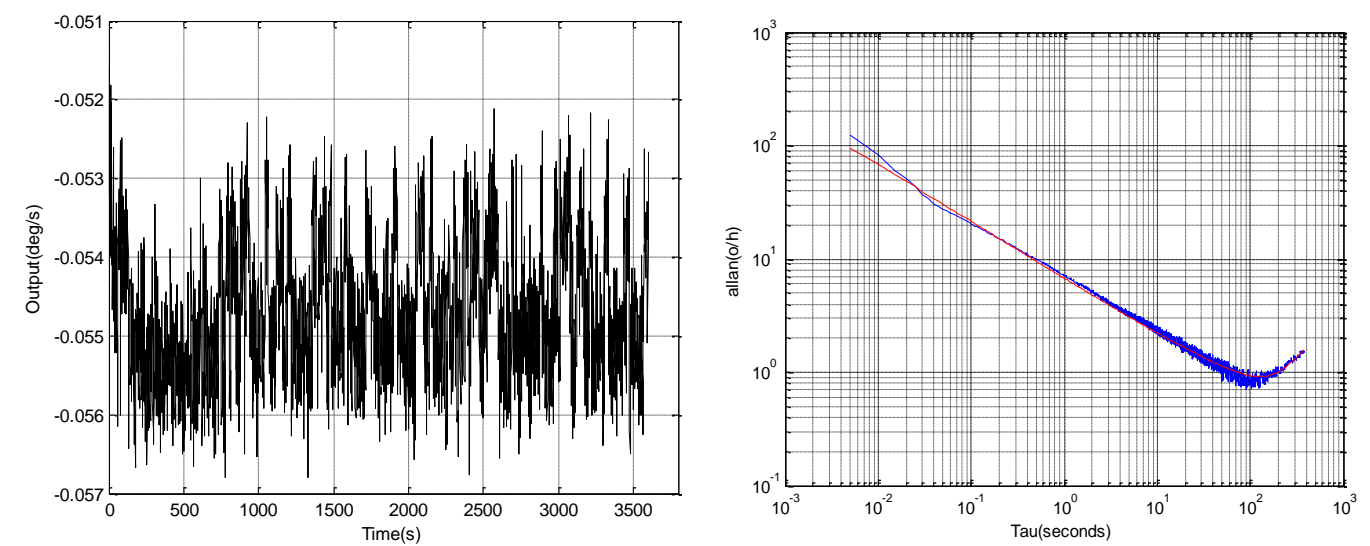

Fig. 6. Zero-rate output of a SiVG Fig.7 Allan variance analysis result.

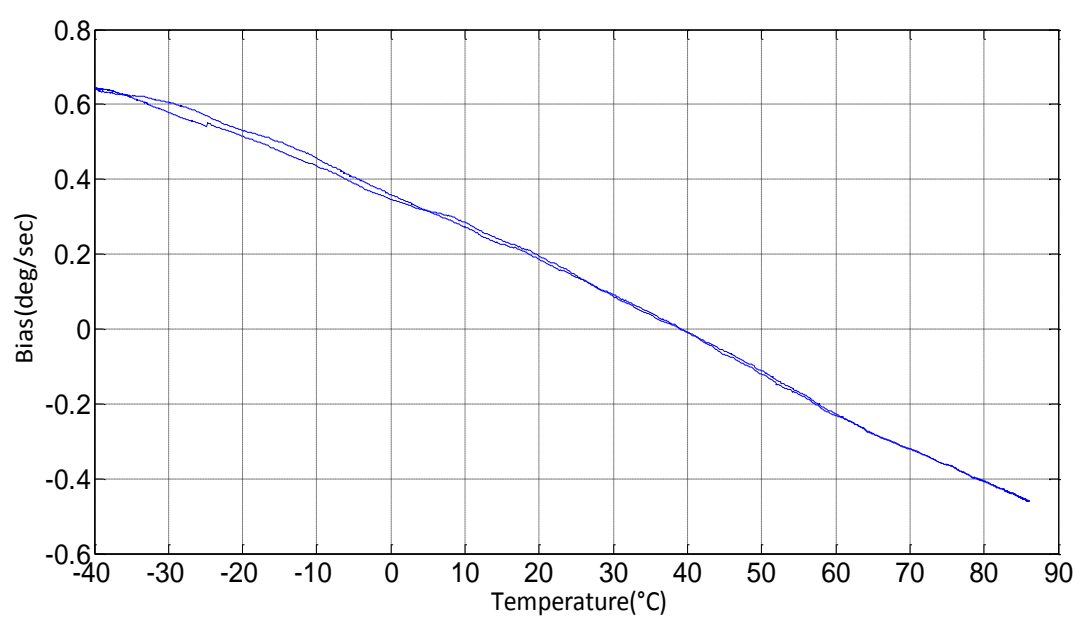

Fig. 8. Temperature hysteresis loop of the SiVG 
Fig. 8 shows the temperature hysteresis loop of the SiVG. The uncompensated zero-rate output is recorded while temperature was varied from $+20^{\circ} \mathrm{C}$, to $+85^{\circ} \mathrm{C}$, to $-40^{\circ} \mathrm{C}$, back to $+20^{\circ} \mathrm{C}$. From the data, we obtain that the temperature hysteresis is about $0.03 \mathrm{deg} / \mathrm{s}$.

Table 1 is the typical performance of the SiVG that we have measured.

Table 1. Typical Performance of the SiVG

\begin{tabular}{|c|c|c|}
\hline Parameter & Unit & Typical value \\
\hline Measurement Range & $\mathrm{deg} / \mathrm{sec}$ & \pm 300 \\
\hline Scale factor Repeatability & $\operatorname{pap}(1 \sigma)$ & $<100$ \\
\hline Scale factor Nonlinearity & $\operatorname{pap}(1 \sigma)$ & 200 \\
\hline Resolution & $\mathrm{deg} / \mathrm{sec}$ & 0.003 \\
\hline Bias Repeatability & $\operatorname{deg} / \mathrm{hr}(1 \sigma)$ & 5 \\
\hline Bias stability & $\operatorname{deg} / \mathrm{hr}(1 \sigma)$ & 5 \\
\hline Bias( In-Run Stability) & $\mathrm{deg} / \mathrm{hr}$ & 1 \\
\hline Bias Static g Sensitivity & $\mathrm{deg} / \mathrm{hr} / \mathrm{g}(1 \sigma)$ & $<10$ \\
\hline Bias $g^{2}$ Sensitivity & $\mathrm{deg} / \mathrm{hr} / \mathrm{g} \wedge 2(1 \sigma)$ & $<1$ \\
\hline Angle Random Walk & $\mathrm{deg} / \sqrt{\mathrm{hr}}$ & 0.06 \\
\hline Temperature hysteresis & $\mathrm{deg} / \mathrm{sec}$ & $<0.05$ \\
\hline Bandwidth & $\mathrm{Hz}$ & 80 \\
\hline
\end{tabular}

\section{SRA}

Operational Principle. The SRA is shown as a schematic in Fig.9. The SRA structure can be divided into three major components, a proof mass, micro levers and DETFs. Two DETFs are joined via micro levers to the proof mass. The proof mass is constrained to move along the y-axis by four flexures which are linked to the frame mounted on the silicon substrate by four anchors. When acceleration along the input axis is applied to the device, the force from the proof mass is magnified by micro levers and then transferred to the DETFs. This input causes an axial load, either tension or compression in the DETFs, which produces a measurable natural frequency shift of the two resonators. The output of SRA is the differential frequency variation of the two resonators proportional to the input acceleration.

The SRA die is also fabricated with SOI processing and wafer-level vacuum packaged as described in Section 2. Fig.10 (a) and (b) show the SEM of the mechanical structure and the view of the wafer-level vacuum packaged SRA die. The proof mass of the SiVG is about $2 \mathrm{~mm} \times 2 \mathrm{~mm}$ in size, the natural frequency of the DETF resonator about $20-30 \mathrm{kHz}$ and the amplification factor of the micro level about 25 . The overall sensitivity of the SRA is about $250 \mathrm{~Hz} / \mathrm{g}$. Fast non-dominated sorting.

Signal Processing. Fig. 11 illustrates the overall block diagram of the SRA system. The DETF resonator works as a high Q frequency selective network and embedded in an oscillation loop. The oscillation sustaining circuit includes a front-end amplifier, a feedback driving circuit and an automatic amplitude control circuit with chopper. The Frequency measurement circuit receive the oscillator signal and reshape it to square wave, then multiple the signal frequency 128 times by a phase locked loop and give out the frequency by a digital counter. The noise of the frequency measurement circuit is proved below the oscillation signal noise.

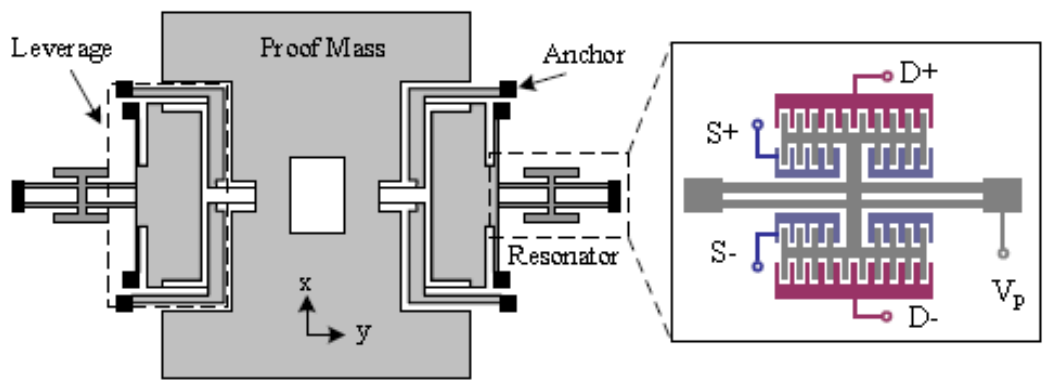

Fig. 9. Schematic of the SRA. 


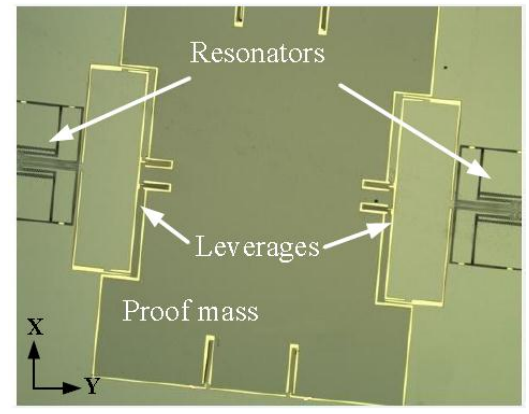

(a)

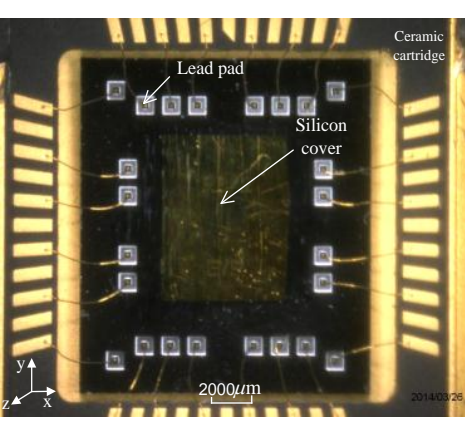

(b)

Fig. 10. (a). SEM of the mechanical structure (b) View of the SRA die.

Performance Testing. Fig.12 is a 32-position static calibration results of $\pm 1 \mathrm{~g}$ of a SRA. By using a linear fitting, the scale factor is $258.9 \mathrm{~Hz} / \mathrm{g}$ with $57.6 \mathrm{ppm}$ nonlinear error in this case.

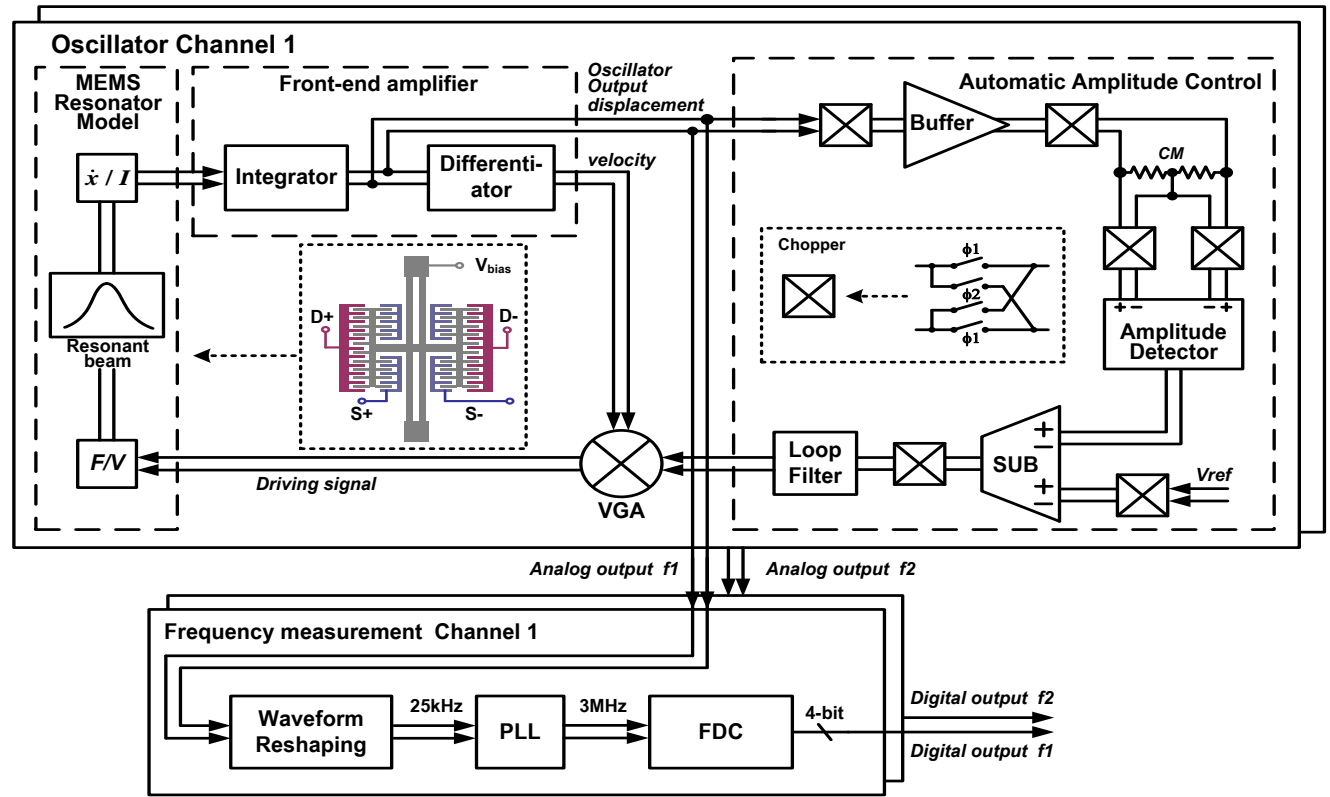

Fig.11 Schematic diagram of SRA signal processing.

The test SRA is mounted on a centrifuge to calibrate the input-output relation in the range of $\pm 45 \mathrm{~g}$. Fig. 13 shows the relative error between the linear fitted values and the output, which is within 50ppm.

Fig.14 is the measured output spectrum density of the accelerometer under no input. It shows that a white noise PSD is $1.2 \mu \mathrm{g} / \sqrt{\mathrm{Hz}}$, which is equivalent to a velocity random walk coefficient of $7.2 \times 10^{-4} \mathrm{~m} / \mathrm{s} / \sqrt{ } \mathrm{hr}$.

Fig. 15 shows the Allan Variance of the frequency expressed in $\mu$ g calculated from the bias data to evaluate bias-instability of the SRA . The measured bias-instability is about $0.5 \mu \mathrm{g} @ 10 \mathrm{~s}$. The low bias instability is achieved by minimizing the flicker noise in the amplitude control circuit by chopper stabilization and loop filter. 

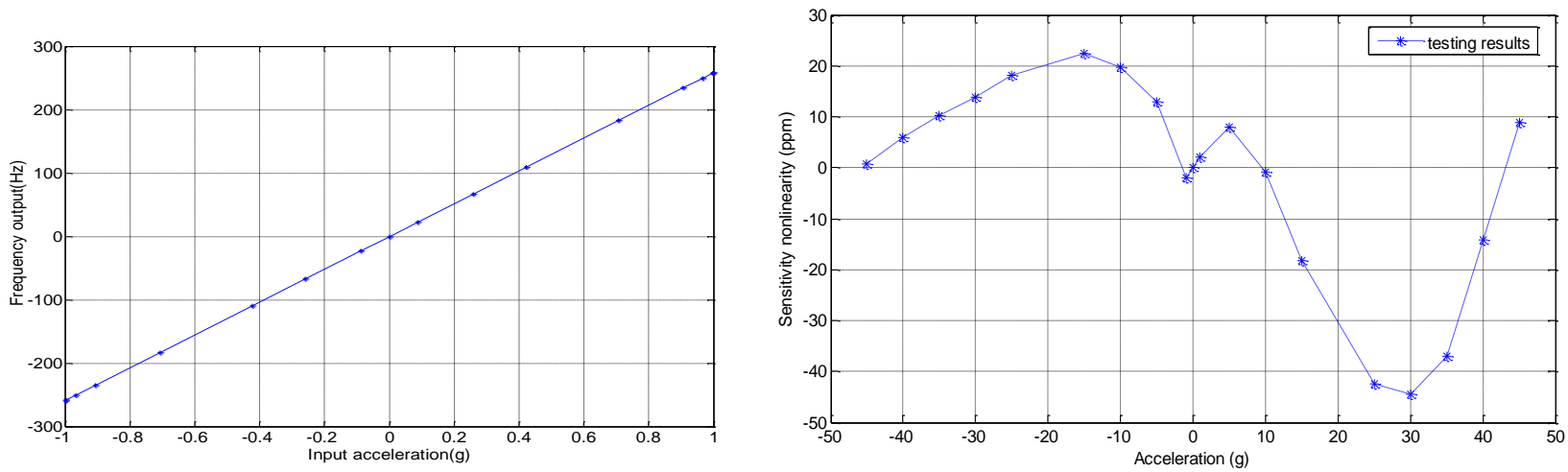

Fig. 12. 32-position test of $\pm 1 \mathrm{~g}$ of a SRA. Fig. 13. Relative error between the linear fitted values and the output.

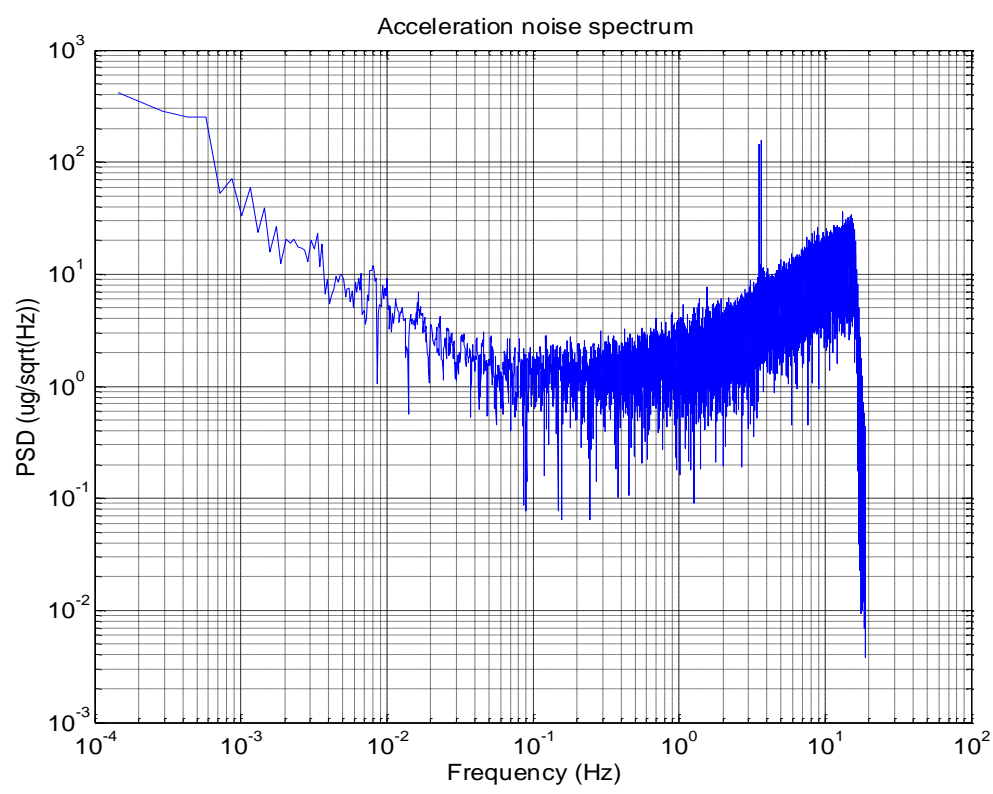

Fig. 14. Measured acceleration noise spectrum.

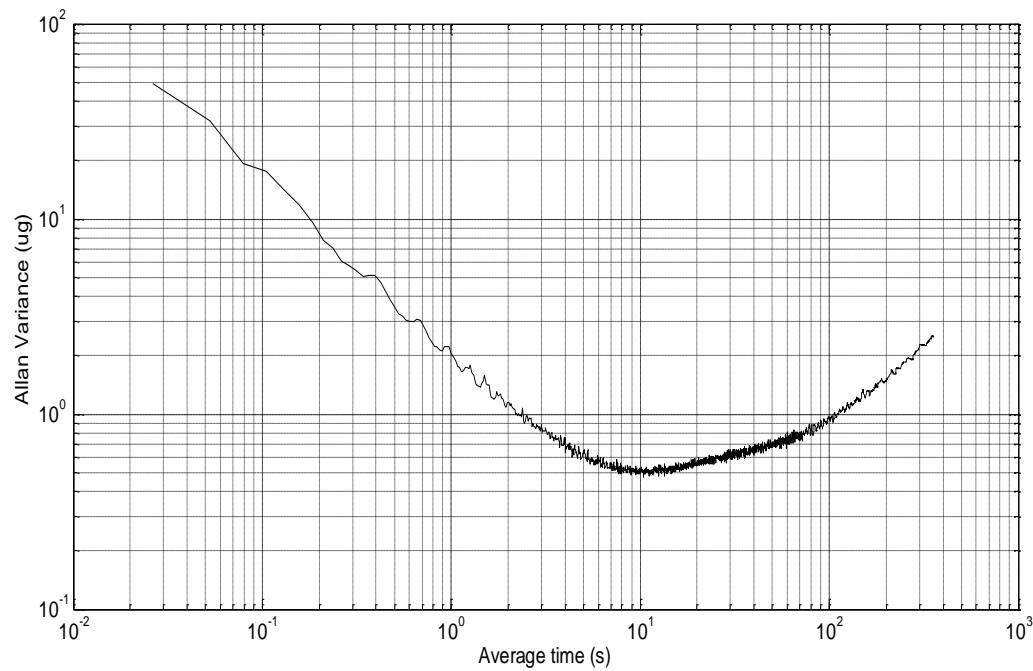

Fig. 15. Allan variance plot.

Table 2 is the typical performance of the SRA that we have measured.

Table 2. Typical performance of the SRA

\begin{tabular}{|l|r|r|}
\hline Parameter & Unit & $\begin{array}{l}\text { Typical } \\
\text { Value }\end{array}$ \\
\hline Measurement Range & $\mathrm{g}$ & \pm 45 \\
\hline
\end{tabular}




\begin{tabular}{|l|r|r|}
\hline Scale factor & $\mathrm{g} / \mathrm{Hz}$ & $\sim 250$ \\
\hline Nonlinearity $\mathrm{k}_{2}$ & $\mu \mathrm{g} / \mathrm{g}^{2}$ & 19.0 \\
\hline Nonlinearity $\mathrm{k}_{3}$ & $\mu \mathrm{g} / \mathrm{g}^{3}$ & 0.06 \\
\hline $\begin{array}{l}\text { In-Run Bias } \\
\text { Stability random }\end{array}$ & $\mathrm{mg} / \mathrm{s} / \sqrt{\mathrm{h}}$ & 0.5 \\
\hline $\begin{array}{l}\text { velocity } \\
\text { walk }\end{array}$ & $\mathrm{Hz}$ & $>1000$ \\
\hline Bandwidth & & \\
\hline
\end{tabular}

\section{Summary}

MEMS Inertial Technology Research Center at NUST are now actively engaged in the development of higher tactical-grade SiVG with a bias stability $(1 \sigma)$ of $1 \mathrm{deg} / \mathrm{hr}$, and inertial-grade SRA with a bias stability $(1 \sigma)$ of $1 \mu \mathrm{g}$. Meanwhile, we also devote to improve environment adaptability of the SiVG and the SRA.

\section{Acknowledgements}

The authors wish to thank Dr. Yongjun Yang and his goup at the 13th Research Institute of China Electronic Technology Group Corporation for their support.

\section{References}

[1] Marc S. Weinberg, and Anthony Kourepenis, Error sources in in-plane silicon tuning-fork mems gyroscopes, Journal of Microelectromechanical Systems, Vol. 15, No. 3, June 2006

[2] A. El-Sayed et al., "A Self-Clocked ASIC Interface for MEMS Gyroscope with $1 \mathrm{~m} \% \mathrm{~s} / \sqrt{\mathrm{Hz}}$ Noise Floor", pp. 1-4, Custom Integrated Circuits Conference (CICC), 2011.

[3] R. Hopkins, J. Miola, R. Setterlund, B. Dow, W. Sawyer, The silicon oscillating accelerometer: a high-performance MEMS accelerometer for precision navigation and strategic guidance applications, TECHNOLOGY DIGEST, (2006) 4.

[4] L. He, Y. P. Xu and M. Palaniapan, "A CMOS Readout Circuit for SOI Resonant Accelerometer with 4- $\mu$ g Bias Stability and $20-\mu \mathrm{g} / \sqrt{ } \mathrm{Hz}$ Resolution," Solid-State Circuits, IEEE Journal of , vol.43, no.6, pp.1480-1490, Jun. 2008. 